\title{
Effect of multifunctional coatings on the moisture transport properties of ETICS
}

\author{
Giovanni Borsoi $^{\mathrm{a}}$, Renata Roncon ${ }^{\mathrm{b}}$, João Parracha ${ }^{\mathrm{a}, \mathrm{c}}$, Inês Flores-Colen ${ }^{\mathrm{a}}$, Rosário Veiga ${ }^{\mathrm{c}}$ \\ ${ }^{a}$ CERIS, Instituto Superior Técnico, University of Lisbon, Lisbon, Portugal \\ ${ }^{b}$ DeCivil, Instituto Superior Técnico, University of Lisbon, Lisbon, Portugal \\ ${ }^{c}$ National Laboratory for Civil Engineering, Lisbon, Portugal
}

\begin{abstract}
External Thermal Insulation Composite Systems (ETICS) have been extensively applied for increasing the thermal insulation in both new and retrofitted constructions. These systems, however, can present several anomalies, due to their constant exposure to weathering agents and anthropic factors, which can lead to cracks and stains. The application of protective coatings aims at minimizing these anomalies and increasing the durability of ETICS. In fact, a proper maintenance of these systems is necessary to achieve a durable service life.

In this paper, we study the effect of multifunctional coatings when applied on ETICS. Commercially available paints, with improved (i.e. hydrophobic, antibacterial and self-cleaning) properties were selected and compared, evaluating the effect of these treatments on the moisture transport properties of ETICS.
\end{abstract}

Keywords: ETICS; Coatings; Multifuctionality; Moisture transport properties; Durability

\section{Introduction/Background}

The EU has set new rules for energy efficiency, both in new and retrofitted buildings, with the objective of achieving a nearly zero energy built environment by 2050 [1]. In this context, External Thermal Insulation Composite Systems (ETICS), also identified as External Wall Insulation (EWI, in the UK), can significantly improve the thermal performance and energetic efficiency of buildings [2]. These systems, however, can suffer damages, due to their constant exposure to weathering agents and anthropic factors, which can lead to e.g. cracks and stains. Water is generally the major cause of decay, since it can trigger physical-mechanical-chemical degradation and biocolonization. For these reasons, the application of hydrophobic and multifunctional treatments can improve ETICS service life, by reducing the wettability and the water absorption of the systems while conferring them additional functionalities. Acrylic and silicon compounds are among the most used film-forming hydrophobic products, generally providing suitable effectiveness and having good compatibility with ETICS top coat. However, the durability of these coatings can be affected by weathering, UV radiation, extreme $\mathrm{pH}$ conditions or pollutants [3], altering their water-repellent properties and thus long-term effectiveness.

In this study, the performance of three different multifunctional paints with hydrophobic features was analysed, when applied on ETICS, with the aim of assessing the compatibility, effectiveness, and durability of these multifunctional products, which have also self-cleaning and biocide properties. The moisture transport properties (water absorption by capillarity, drying kinetics, water vapor permeability, and contact angle measurement) of treated and untreated specimens were evaluated. The durability of the treatments was also tested, through artificial aging tests (freeze-thaw and heat-cold cycles).

\section{Materials and Methods}

ETICS specimens, composed of EPS insulation board, a finishing render with a cementiceous basecoat and an acrylicbased top coat, were studied. Among multifunctional coatings, two acrylic-based (H1, H3) and one siloxane-based (H2) paints were selected (Fig. 1a). These products have also antibacterial (biocide additive) and self-cleaning (photocatalytic additive, i.e. titanium oxide nanoparticles) features. Capillarity water absorption tests (according to EN 15801), drying kinetics (EN 16322), water vapor permeability (ETAG004 [4]), contact angle (by sessile drop technique, i.e. 
Axisymmetric Drop Shape Analysis) were carried out in threefold in prismatic specimens; accelerated aging tests were adapted from EN 1015:21 (i.e. heat-cold and freeze-thaw cycles).

\section{Results}

Results showed that the multifunctional paints moderately decrease the capillarity water absorption coefficient (WAC) (around 25-35\% for all treatments), being H3 (acrylic-based paint) the less water absorbent treatment (Fig. $1 \mathrm{~b}$ ); after artificial aging, there is a significant WAC reduction in untreated specimens (up to $45 \%$ compared to unaged specimens), which can be attributed to the obstruction or destruction of capillarity pores (of the ETICS base and/or top coat) due to freeze-thaw cycles. In fact, additional hydration reactions of the cement of the basecoat can induce a reduction of the capillary pores of the basecoat, or, conversely, some of the capillary pores can be modified over capillary dimension. However, even after artificial aging, the specimens treated with hydrophobic products continue to repel water slightly more than aged, untreated specimens. Concerning the contact angle test, H2 (siloxan-based) provides the higher water repulsion and thus lower interfacial tension; a similar trend is observed after artificial aging, and the general $\theta$ increase confirms that the aging cycles possibly alter the microstructure (significant porosity reduction) of the specimens. It should be said that $\theta>90^{\circ}$ (i.e. hydrophobic surface) is observed only in the case of the aged H2 specimen (Fig. 1c). The inclusion of significant percentages of $\mathrm{TiO}_{2}$ (which generally provides hydrophilic properties, thus increasing wetting) can interfere in those values. In fact, water repellence takes place on low-energy surfaces, where the attraction between the molecules of the solid and liquid interface is weak [5], and conversely, $\mathrm{TiO}_{2}$ has a high energy surface. Regarding the drying phase governed by water vapour transport, $\mathrm{H} 2$ slightly decreased the drying capacity of the treated specimens $(5 \%)$, whereas the acrylic-based treatments $(\mathrm{H} 1, \mathrm{H} 3)$ have a higher impact (higher delay, especially in the case of $\mathrm{H} 3-70 \%$ ); the general decrease of DR2, justified also by the lower amount of water absorbed, is confirmed after artificial aging. The results of the water vapor permeability tests confirm that the hydrophobic products slightly decrease the specimens permeability (13-24\%); after aging, $\mathrm{H} 3$ shows a higher reduction of the WVP (i.e. higher $\mu$ ), if compared to the unaged specimen.
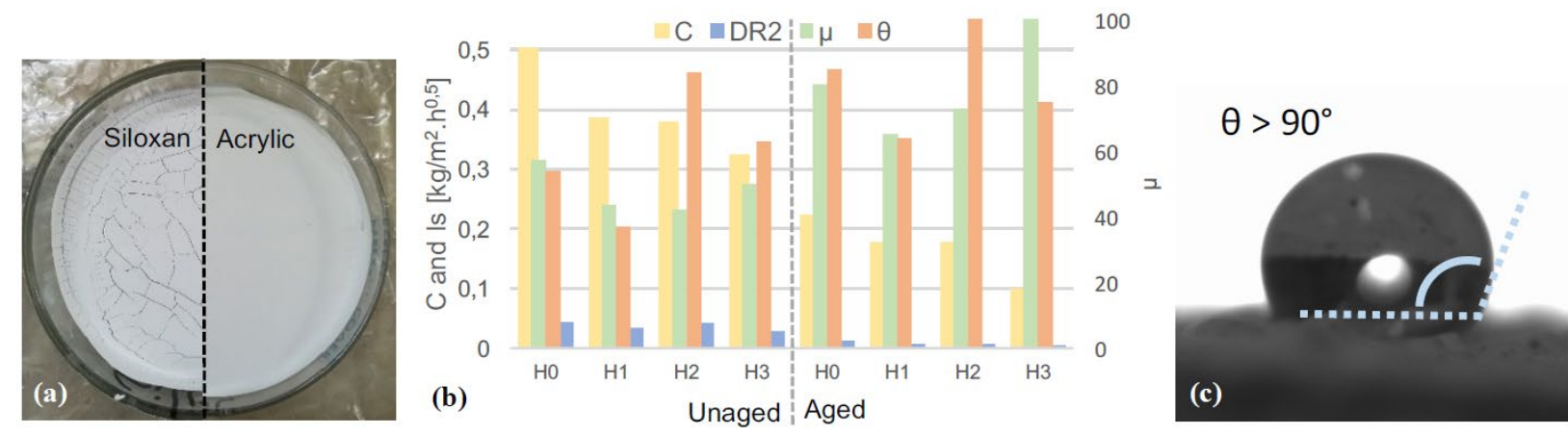

Figure 1. (a) Dry residue of H2 (siloxane) and H3 (acrylic-based paint); (b) Moisture transport properties of untreated and treated (unaged and aged) of specimens H0, H1, H2, H3 (C: water absorption coefficient by capillarity; DR2: drying rate in the vapor phase; $\mu$ : water vapor diffusion resistance factor; $\theta$ : contact angle); (c) contact angle of aged specimen treated with $\mathrm{H} 2$.

\section{Conclusions}

All products decreased the capillary water absorption, slightly delayed the drying rate, and somewhat decreased the water vapor permeability. Contact angle measures confirm that siloxane-based paint (H2) provides the higher surface hydrophobicity, still providing a (physical) compatibility with the treated ETICS (similar drying rate and water vapor permeability). After aging, the hydrophobic products still show a reasonable effectiveness. However, acrylic coating $\mathrm{H} 3$ retards slightly more the water vapor transport and its use is thus not recommendable on ETICS. Additionally, the ETICS systems underwent an alteration (possible modification of the porosity) due to the aging cycles. The addition of $\mathrm{TiO}_{2}$ (present in all coatings) possibly interferes in the alteration of the wetting of the treated surface.

\section{References}

[1] Energy Performance of Building Directive (EPBD) (2010). Directive 2010-31-EU of the European Parliament and of the Council. Official Journal of the European.

[2] Barreira, E. \& P. de Freitas, V. (2013). Experimental study of the hygrothermal behaviour of External Thermal Insulation Composite Systems (ETICS). Building and Environment, 63, 31-39.

[3] Borsoi, G., Esteves, C., Flores-Colen, I., Veiga, R. (2020). Effect of Hygrothermal Aging on Hydrophobic Treatments Applied to Building Exterior Claddings. Coatings, vol. 10, p. 363, 2020.

[4] EOTA (2013). Guideline for European technical approval of external thermal insulation composite systems (ETICS) with rendering (ETAG 004). Brussels.

[5] Leelamanie, D.A.L., Karube, J., Yoshida, A. (2008). Characterizing water repellency indices: Contact angle and water drop penetration time of hydrophobized sand. Soil Science and Plant Nutrition, 54, 179-187. 